\title{
La concertación de grandes actuaciones urbanas en España
}

Félix Arias Goytre *

En este trabajo se realiza una sistematización y comparación de las nuevas formas de concertación que se están utilizando en el desarrollo de distintos tipos de actuación urbana estratégica. Se revisa la experiencia reciente de las ciudades españolas en las dos últimas décadas, con especial mención a las Estrategias Territoriales de la Comunidad de Madrid y a la Política de Ciudades incluida en el Plan Director de Infraestructuras del Ministerio de Fomento, en las que el autor ha desarrollado su trabajo profesional.

\section{El marco actual de las políticas urbanas}

Las políticas dominantes hoy en día pretenden limitar el campo de juego de las políticas urbanas a medidas de desregulación de los costes sociales y ambientales, con el fin de favorecer la competitividad empresarial, en especial de las empresas transnacionales y de alto valor añadido, como forma de fomentar la creación de empleo. Se deja la resolución de muchos daños «colaterales» sociales y ambientales, producidos por la desregulación, a las posibilidades redistributivas que supuestamente permitirá la mejor marcha de la economía. Sin embargo, los sectores sociales más desfavorecidos no se benefician de la generación de empleo, aumentando la desigualdad social, y muchos efectos ambientales son de difícil reparación.
Pero la globalización es un conjunto más complejo de procesos sociales, políticos, culturales, etc., que la mundialización económica que nos presenta el pensamiento único, y sus detractores más radicales, produciéndose contradicciones entre procesos y entre escalas (locales, nacionales y globales) (CASTELLS, 1997).

A medio plazo, serán competitivos los países y ciudades que favorezcan el aumento global de la productividad, mediante tecnologías para la reducción del consumo de energía y de materiales, ambos necesarios para lograr un desarrollo sostenible, y los que impulsen procesos urbanos que fomenten la cohesión social. Los que fomenten situaciones de degradación del factor trabajo crearán desigualdad, exclusión y malestar social, con el consecuente incremento de las luchas sociales para mejorar los salarios y aumentar los impuestos que financien el desarrollo de servicios públicos; sin contar los efectos negativos que la precarización económica de amplios sectores tiene sobre la demanda interna.

Como consecuencia del enfoque de la globalización, orientado a fomentar una competitividad económica mal entendida, las propuestas territoriales que se han divulgado más en las últimas décadas se basan generalmente en el desarrollo de grandes infraestructuras y en operaciones urbanísticas para actividades económicas de elite. Al mismo tiempo, la cohesión (empleo, integración social, derechos ciudadanos, etc.) en las zonas perdedoras de las ciudades y la sostenibilidad ambiental (local y global) no se han abordado como objetivos estratégicos territoriales, atendiéndose tan sólo con políticas de acompañamiento para la atención de los casos más graves de exclusión y de degradación ambiental. 


\section{Una gestión urbana integrada y estratégica}

Para afrontar los problemas actuales y venideros es necesario plantear la eficiencia global de los ecosistemas urbanos y de sus relaciones con el entorno próximo o lejano, para sustentarse; no se pueden tratar independientemente los objetivos de competitividad, cohesión y sostenibilidad.

Asimismo, hay que considerar que la organización física, social y funcional de la ciudad contribuye a una mayor o menor calidad de vida y productividad del sistema en su conjunto, y de los hogares y empresas que lo habitan, así como a una mayor o menor depredación del capital humano y natural.

Los objetivos estratégicos del desarrollo urbano sostenible, en las ciudades europeas (CCE, 1998), se están concretando básicamente en:

- Creación de empleo con especial atención a los jóvenes, mujeres y parados de larga duración.

- Integración social con atención a la promoción de las identidades locales; la pluriculturalidad y la integración de inmigrantes.

- Sostenibilidad ambiental con especial atención a la gestión de la demanda, el transporte, la producción industrial y el turismo.

- Desarrollo de mecanismos de democracia participativa para la toma de decisiones y de gestión cooperativa de las actuaciones públicas.

Para la definición de una estrategia que responda a estos cuatro objetivos se han de promover mecanismos de debate amplio con los agentes sociales y procesos de concertación para formular los objetivos concretos de la ciudad o barrio, los procesos a impulsar, actuaciones a desarrollar y mecanismos de responsabilización de los agentes sociales (Administraciones, iniciativa privada, tercer sector y movimientos sociales).

Las Administraciones locales y territoriales más avanzadas empiezan a comprometerse en el impulso de estos procesos de gestión, facilitando los medios y organización adecuados y liderando las actuaciones estratégicas en cooperación con los agentes que se han de implicar para el logro de los objetivos de un desarrollo urbano sostenible.

Lamentablemente son aún escasas las experiencias emergentes en este sentido y, en cambio, como se verá a continuación, abundan las actuaciones, especialmente entre las grandes operaciones urbanas, en las que se actúa con un criterio inmobiliario e infraestructural, sin incorporación de objetivos sociales ni ambientales adecuados y sin contar con los agentes ciudadanos que pueden defenderlos.

\subsection{Un planeamiento orientado a una actuación integral sobre los problemas}

El reconocimiento de la complejidad de los problemas y de la necesidad de contemplar de forma integrada distintos aspectos de la realidad, campos de actuación y agentes diversos está revolucionando el planeamiento.

El planeamiento municipal se va haciendo cada vez más:

- Estratégico, seleccionando los temas y prioridades de actuación, integrando sectores sobre la base de las caracterís. ticas de los problemas.

- Director, dando criterios para la coordinación de los planes y formas de actuación, sectoriales y locales.

- Continuo y evaluativo, realizando auditorías sobre la evolución de los sistemas urbanos o algunos de sus elementos, los impactos de las actuaciones, etc.

El éxito de la política municipal va a depender de la capacidad que se tenga para analizar los problemas, definir las necesidades de actuación implicando a las partes afectadas y disponer de instrumentos de ejecución adecuados.

En distintos momentos se van poniendo de moda técnicas que abordan con mayor o menor destreza el planeamiento municipal, con el objetivo de actuar más integralmente frente a los objetivos de prosperidad, cohesión, sostenibilidad y gobernabilidad. Hoy en día los sistemas de planeamiento más en boga son, por un lado, los Planes Estratégicos, que surgen desde el planeamiento de las empresas, orientados a las actuaciones de desarrollo de actividades económicas y que van ampliando su temática y, por otro, las Agendas 21, que parten del enfoque del desarrollo sostenible, desde las componentes ambientales y sociales.

También se observa una tendencia de los planes sectoriales, especialmente los de usos de suelo y los de transporte, a integrar otra temática en sus planteamientos, desarrollando estrategias territoriales o de transporte, que se formulan con una visión global de las ciudades y establecen criterios para otros instrumentos de actuación, aparte de los suyos propios de gestión urbanística, promoción de suelo, actuaciones de infraestructuras, etc.

Como se verá, es interesante analizar el proceso de trabajo que han seguido, antes y después del «Plan», las ciudades que han aplicado con mayor éxito planes de uno u otro tipo. Al hacerlo se observa que el plan, en sí, no suele ser el desencadenante de los nuevos valores, sino que ha sido un instrumento dentro de unos procesos en marcha en la gestión y la política municipal. 
Las ciudades que han abordado una nueva forma de planeamiento sin poner en marcha procesos políticos adecuados, de cooperación interna, con otros agentes y con la sociedad civil, han realizado planes que apenas han tenido una incidencia real en la visión estratégica y directora de su desarrollo, convirtiéndose con el tiempo en documentos que no han tenido continuidad como mecanismos gestores.

La cuestión fundamental para abordar adecuadamente la nueva problemática de las ciudades es la voluntad política de modificar la forma de intervención pública y la propia organización administrativa, en la línea estratégica, directora, evaluadora y cooperativa que venimos enunciando. Esta constatación no significa que para actuar con nuevos criterios en los municipios haya que esperar a establecer un nuevo marco legal, pero sí que hay que empezar a establecer fórmulas cooperativas entre los sectores administrativos y con el conjunto de los agentes sociales.

En España, la escasa cultura política sobre las vías para un desarrollo más sostenible y la inercia de la estructura sectorial de las Administraciones, están provocando que se asimilen de forma incorrecta las nuevas formas de planeamiento y gestión, confundiendo las políticas integrales con la agrupación de políticas sectoriales (departamentales) en paquetes o planes multisectoriales (ARIAS, 1998a).

\subsection{Una gestión concertada y participativa}

La cuestión no es tanto la elección entre tipos de planeamiento, como crear la capacidad para desarrollar diagnósticos y objetivos adecuados orientados al desarrollo urbano sostenible y, para ello, es necesario tener la voluntad política de desarrollar un sistema de gestión que se base en la interacción institucional y social, de tal forma que los distintos instrumentos que se utilicen interaccionen entre sí sin sesgar las actuaciones hacia ninguna visión sectorializada de la ciudad (BORJA, 1996).

La realidad urbana es muy compleja, y los reguladores con los que se trata de intervenir tienen que tener una complejidad «suficiente», como establecen las leyes cibernéticas. Lo fácil es hacer actuaciones sectoriales (más cemento, más subvenciones, más cursillos, etc.), que acaban conduciendo muchas veces a resultados distintos de los objetivos propuestos (generan más tráfico, desempleo en los mismos barrios de siempre, más contaminación en la atmósfera y en los acuíferos, etc.) (NAREDO, 1996; RuEDA, 1996; PraTs, 1996; CNH, 1996a).

Sólo se conseguirá una mayor integralidad de las actuaciones si se parte de los problemas y se buscan soluciones con los afectados; considerando siempre también la escala local o de barrio. Las propuestas tienen que sustentarse en los hábitos cotidianos de los usuarios de la ciudad (producción, consumo, ocio, etc.), logrando una corresponsabilidad para introducir cambios culturales que se han de asumir por todos, frente al fomento de los hábitos de consumo que promueve el mercado cuando sólo busca el aumento de las ventas.

La manera de ir cambiando las cosas es ir haciéndolas, reflexionar sobre los resultados y aprender a mejorarlas. Para ello es necesaria una toma de decisiones compartidas, con conocimiento del por qué y para qué, de forma que la sociedad empiece a asumir que la ciudad es un proyecto sobre el que pueden incidir y no algo ajeno que escapa a su control. Si las nuevas propuestas para el desarrollo sostenible no cuentan con la complicidad de la sociedad, seguirán siendo algo impuesto y seguirán fracasando (Rodríguez VILLASANTE, 1996; CHANAN, 1997).

Si bien muchas de las medidas necesarias, para poder abordar políticas con vistas a un desarrollo sostenible y solidario, corresponden a organismos mundiales y estatales (ARIAS, 1996, 1998c), también hay medidas importantes que desarrollar desde las regiones y en las ciudades (ARIAS, 1998a, 1999b).

La ciudad, y sus barrios, son los espacios que permiten la focalización de las políticas en los problemas, y la dinamización y protagonismo de los afectados que, si no, se ven sobrepasados por las grandes políticas destinadas a los grupos de poder dominantes que ignoran las redes locales existentes (sociales y empresariales), sus necesidades y sus posibilidades de articulación con otras redes exteriores a la ciudad.

Los nuevos pactos sociales necesarios para impulsar un desarrollo urbano más sostenible y solidario han de apoyarse en un mayor consenso en las estrategias a seguir por la iniciativa pública, con el apoyo y participación de la sociedad civil, la iniciativa privada y el tercer sector. Para ello hace falta una reorganización de las estructuras de poder:

- Devolviendo poder a la sociedad civil, promoviendo la responsabilidad de los individuos en el ejercicio de sus derechos y deberes, y el fortalecimiento del tejido social como mediador entre las personas y los gobiernos.

- Abriendo los gobiernos como espacios en los que reconciliar intereses entre los afectados y orientar las políticas y proyectos, mediante el debate sobre objetivos y alternativas de la política.

- Concertando marcos de actuación entre los gobiernos de distinto nivel, superando las luchas por competencias supuestamente excluyentes para desarrollar una concepción incluyente de gobierno cooperativo.

Estos principios tienen especial significado en los ámbitos locales de los municipios (barrios y ciudades) donde se pueden arbitrar mecanismos, de toma de decisiones y de seguimiento de la gestión, más próximos al ciudadano en las cuestiones que le son más cercanas (CHANAN, 1997; GEDDEs, 1998; RodRíGUEZ VILLASANTE, 1996). 
A lo largo de esta década se han debatido estos temas en todos los organismos multinacionales que tratan la ciudad, aunque la escasez de políticas activas muestra que el debate tiene aún mucho de retórico (ONU, 1992, 1995, 1996; OCDE, 1996, 1998; Presidencia de la UE, 1995, 1997, 1998; Comisión de las Comunidades Europeas, 1990, 1996 y 1998, y Comité Nacional de Habitat, 1996).

\section{Las grandes operaciones urbanas}

Bajo la denominación de gran operación urbana se suelen contemplar distintos tipos de actuaciones. En las ciudades se desarrollan muchas operaciones que, por su tamaño o contenido, inciden de forma significativa en la estructura y el desarrollo urbano.

Las ciudades españolas maduras, que en general están estancadas demográficamente, sufren, sin embargo, transformaciones sociales importantes (p. ej., tamaño de hogar) y económicas, que generan nuevas necesidades de ordenación del espacio urbano, oportunidades de renovación urbana de algunas zonas y aumento de la demanda total de suelo urbanizado.

Las grandes operaciones se han caracterizado por la intervención del poder público en la ordenación de la ciudad, a través del planeamiento y en muchos casos mediante intervención pública directa o mixta. A lo largo de la historia se han promovido espacios para las instituciones, las grandes actividades industriales y las financieras, grandes ensanches, áreas de renovación, infraestructuras y dotaciones de servicios con capacidad estructurante, etc. Hoy en día, las operaciones más publicitadas son las que desarrollan los nuevos espacios, o barrios, del poder financiero, empresarial e institucional, que surgen ante los nuevos requerimientos de edificación, localización, conexiones e imagen, que plantean estas actividades.

La orientación dada por las políticas urbanas neoliberales de las últimas décadas a estas actuaciones ha creado un estereotipo de operaciones estrella que se justifican como necesarias para atraer a las ciudades inversiones de empresas transnacionales y que sirven de apoyo a campañas de marketing político. Son operaciones que desarrollan grandes infraestructuras y actuaciones urbanísticas para actividades económicas de alto valor añadido, concibiéndose como espacios exclusivos a veces fuertemente segregados de su entorno.

Ante la falta de capacidad que suelen tener los centros urbanos existentes para acoger estas demandas, se desarrollan actua- ciones en localizaciones que pretenden crear «nueva centra. lidad», en lugares topológicamente centrales o en nodos periféricos de comunicaciones. Las actuaciones se conciben con un acompañamiento de otros usos (comerciales, residenciales, ocio, etc.) capaces de pagar altos precios por situarse en estos conjuntos inmobiliarios de centralidad e imagen.

Sin embargo, hay otras muchas actuaciones de fuerte impacto para las ciudades, actuaciones que promueven nuevos barrios, renuevan barrios en decadencia introduciendo nuevas actividades complementarias, reurbanizan espacios urbanos abandonados mejorando la calidad y servicios del entorno, gestionan las infraestructuras para mejorar la accesibilidad, etc.

Unas y otras desencadenan procesos que tienen fuertes impactos en el conjunto de la ciudad y en los barrios de contacto, dependiendo de su grado de integración física, complementariedad socioeconómica y complejidad funcional, así como de las características de los agentes que se apropien de las rentas diferenciales generadas y de la utilización que se haga de éstas con fines especulativos y/o redistributivos. El plan de la ciudad y el proyecto de la operación son, por lo tanto, elementos fundamentales que pueden utilizarse en beneficio de distintos objetivos y agentes, como se ha enunciado en los apartados anteriores.

\subsection{Los diversos tipos de grandes actuaciones}

Con objeto de entender mejor las diferentes características de las grandes actuaciones que se están produciendo en nuestras ciudades, es útil clasificarlas por el objeto fundamental de la actuación. Aunque las grandes operaciones suelen tener un contenido complejo, que incluye distintos elementos urbanos, hay uno que es la base u origen de la actuación, y que determina las principales características en cuanto a usos, aprovechamientos, localización, y también en cuanto a las formas de gestión para la ejecución y explotación.

\section{A. Grandes infraestructuras de transporte en la ciudad}

Los grandes proyectos de este grupo pueden incluir la realización o modernización de vías (carreteras o ferrocarriles), la extensión de puertos (con abandono del puerto viejo) o aeropuertos (a veces se abandonan antiguos aeródromos poco útiles en el nuevo sistema aeroportuario), y la ampliación (extensión y nuevos usos) de estaciones ferroviarias, portuarias o aeroportuarias.

La localización y características de cada actuación vienen determinadas por la concepción del modelo de transporte interregional/internacional y por el modelo territorial/transporte 
local. Son actuaciones de concepción y gestión pública, que se asientan en terreno público existente o de nueva adquisición, aunque en algunos casos se produzcan concesiones al sector privado para la ejecución y/o explotación.

El fuerte impacto que producen en la movilidad urbana, los flujos de viajeros y mercancías, convierten las estaciones en potentes intercambiadores del transporte local, modificando el mapa de accesibilidad de la ciudad y convirtiéndolas en áreas muy atractivas para la localización de las actividades que buscan lugares de gran accesibilidad. Algunas de estas actividades son complementos beneficiosos para la mejor utilización del sistema de transporte público (comercio, oficinas, servicios, etc.), pero también provocan importantes externalidades negativas ambientales en el entorno (congestión, contaminación, etc.).

Por todo ello, estas actuaciones tienen un fuerte impacto en las rentas diferenciales del suelo de la zona en que se ubican. Según se planifique su desarrollo y entorno, se producirán distintos impactos que provocarán alzas o bajas en los precios inmobiliarios, ocasionándose la degradación o revalorización de distintas zonas. Presentan, por lo tanto, la oportunidad de concebirlas de forma que capten las plusvalías resultantes, para destinarlas a minimizar los impactos negativos y financiar obras y servicios que contribuyan a una redistribución en favor de las áreas más degradadas. Esta característica hace que las actuaciones urbanísticas tiendan a sobrecargarse en su oferta inmobiliaria, para optimizar sus resultados económicos y lograr ingresos para las Administraciones actuantes.

Las operaciones más significativas en nuestro país son:

- Aeropuertos: Los aeropuertos de Barajas (Madrid) y El Prat (Barcelona) están sufriendo la ampliación de sus instalaciones con fuertes impactos sociales y ambientales en su entorno, y están desarrollando zonas logísticas de mercancías y nuevas áreas terciarias en torno a sus terminales de carga y viajeros, al tiempo que se desarrollan intercambiadores con los ferrocarriles urbano y de largo recorrido (Estudios Territoriales, 1992). En ambos casos se han realizado convenios, entre las Administraciones y agencias públicas, para la ordenación de los aeropuertos y su entorno, pero las actuaciones las lleva a cabo la Empresa Estatal de Aeropuertos (AENA) de forma aislada. En 1995 se aprobó un convenio entre los tres niveles de la Administración para coordinar la operación Barajas y crear una sociedad Ciudad Barajas 2000 que desarrollara el entorno de las terminales, pero quedó bloqueada por recelos políticos.

- Puertos: Los grandes puertos están ampliándose en áreas de mayor calado y abandonando el puerto viejo, normalmente próximo al centro de la ciudad, que pasa a destinarse a tráfico urbano, turismo, etc. $\mathrm{Al}$ mismo tiempo, en las nuevas extensiones de los grandes puertos como Barcelona o Valencia se están potenciando zonas logísticas, que gestiona la autoridad portuaria (empresas estatales). El abandono de las áreas centrales por la actividad portuaria está permitiendo recuperar la fachada marítima de las ciudades, siendo el ejemplo más sig. nificativo el de la remodelación del Moll de la Fusta en el centro de Barcelona realizado en 1980, como gran espacio público que da cobijo (de forma semi oculta) al cierre de una gran vía de circunvalación (BusQuETs, 1992; MOPU, 1985; Urbanismo, 1987; Estudios Territoriales, 1992). Posteriormente los muelles exteriores de la zona vieja del puerto han asentado mediante concesiones administrativas un gran centro de ocio y el World Trade Centre, con un sentido urbanístico muy distinto. Otras actuaciones interesantes de recuperación de puertos viejos se han producido en Gijón, Alicante, etc.

- Estaciones ferroviarias e integración de trazados urbanos existentes: Casi todas las ciudades españolas de más de 50.000 habitantes tiene problemas de convivencia entre el FFCC y la ciudad debido a que los trazados del siglo pasado se plantearon para trenes de largo recorrido y no para uso urbano, y a que las ciudades crecieron posteriormente con un mal diseño urbano en el entorno de las vías. Recientemente se han abordado algunas actuaciones importantes de integración ferrocarril ciudad. La primera que se realizó con un nuevo procedimiento de concertación fue la del Pasillo verde ferroviario de Madrid, que afectó a $9 \mathrm{Kms}$ de recorrido urbano y a los terrenos de tres antiguas estaciones de mercancías, que permitieron dotar la zona con nuevos parques, equipamientos, viviendas y oficinas. Esta actuación se diseño mediante un convenio, entre el Ayuntamiento y RENFE, para luego crear un Consorcio que financió las obras (único caso de autofinanciación en nuestro país), con la venta de los terrenos edificables. Posteriormente se han abordado operaciones similares en Bilbao, Oviedo y Jerez, dirigidas por sociedades públicas participadas por todas las empresas y Administraciones públicas afectadas (Estudios Territoriales, 1992; ARIAS, 1999a). Finalmente, en el municipio de Madrid se está ensayando un procedimiento basado en la concesión al sector privado para la remodelación de la estación de Chamartín y su entorno (terrenos de la empresa de Ferrocarriles y del Ayuntamiento), que ha resultando un fracaso, ya que después de siete años la concesionaria y las Administraciones no han llegado todavía a un acuerdo sobre las características inmobiliarias y la inversión que puede asumir la operación.

- Vias urbanas: En los años ochenta se dieron las mejores muestras del diseño de nuevas vías de alta capacidad como nuevos espacios urbanos locales que daban sentido a todo un distrito, en vez de romperlo o degradarlo (MOPU, 1985; Urbanismo, 1987). En Madrid (LEIRA, 1984) las muestras más significativas fueron la Avenida de la Ilustración, que cerraba la circunvalación M30 (autovía) por el Norte con un diseño de gran paseo urbano con bulevares, la remodelación de la gran 
plaza de Atocha (eliminando los pasos elevados), la Gran Vía de Hortaleza (nueva vía en una zona semiconsolidada periférica) o la Avenida de Curtidores en Tetuán (nueva vía en una zona antigua de remodelación). En Barcelona el diseño del paso de la B20 por el Moll de la Fusta, en la fachada de la ciudad vieja al mar, fue el resultado de las luchas vecinales de los setenta y representó el primer proyecto de autovía urbana domesticada, y posteriormente el resto de la B20 realizada a finales de los ochenta con motivo de la Olimpiada desarrolló soluciones de semienterramiento y falsos túneles en función de las características de los barrios (ACEBILLO, 1992, y BUSQUETS, 1992).

\section{B. Grandes equipamientos}

En el nuevo desarrollo de Madrid algunos grandes equipamientos han tenido un importante valor de recualificación urbana, como, por ejemplo, las universidades públicas que se localizaron en la estrategia territorial, recuperando edificios públicos importantes en las ciudades medias del sur de la metrópoli, mediante convenios entre los Ayuntamientos, el Gobierno regional y el Ministerio de Defensa (propietario de terrenos e inmuebles). La pieza de mayor envergadura fue, sin embargo, el Parque de las Naciones, situado entre Barajas y la periferia este de la ciudad, que desde el punto de vista urbanístico es un espacio segregado de la ciudad, eso sí, con un fuerte impacto en las rentas del suelo de las zonas de ensanche próximas que supuso la aparición de nuevos barrios para rentas medias y altas, pero no la recualificación de los barrios en declive de los distritos adyacentes. La operación se inició a principios de los ochenta cuando el Ayuntamiento de Madrid acometió el desarrollo de los nuevos feriales, adquiriendo terrenos y desarrollando mediante una empresa pública municipal: una gran área de oficinas, un parque metropolitano y los recintos feriales con una empresa participada por las organizaciones de empresarios (Urbanismo, 1992).

\section{Promoción de espacio inmobiliario para nuevas actividades económicas: centros financieros y de oficinas, parques tecnológicos, parques empresariales, parques logísticos, etc.}

Las grandes operaciones de infraestructuras enunciadas en el primer apartado incluyen normalmente espacio para actividades económicas; así, por ejemplo, se han incluido en la operación de Bilbao Ría 2000 (AbandoIbarra y Galindo), en Oviedo, en el puerto de Barcelona, y están previstas en Chamartín, Barajas o El Prat. El Parque de las Naciones en Madrid, que se ha mencionado, ha incluido un complejo de oficinas y servicios junto a los Feriales. Por otro lado, la Comunidad de Madrid se planteó en las estrategias subregionales convenidas con los ayuntamientos periféricos de la metrópoli madrileña, el desarrollo de Áreas de Nueva Centralidad, mediante un operador específico (ARPEGIO, S. A., una empresa pública para promoción inmobiliaria) que desarrollaba actuaciones para distintos tipos de actividad económica en el conjunto del área metropolitana (ARIAS, 1989 y 1991).

\section{Operaciones inmobiliarias de explotación comercial: parques temáticos, grandes centros de ocio y comercio, instalaciones deportivas, etc.}

Se trata en este apartado de operaciones de gran escala, dedicadas al ocio o comercio, explotadas por un único operador privado, que se realizan normalmente en suelo privado. Son operaciones que se localizan en áreas periféricas, y se apoyan en el transporte privado. Las más características son los grandes centros comerciales que proliferan por las periferias urbanas y que se localizan tras algún tipo de acuerdo con las autoridades municipales, muchas veces a través de alguna modificación de planeamiento y haciéndose cargo de algunas inversiones de infraestructura de conexión o de la cesión de terrenos para usos públicos. También pueden considerarse en este apartado algunos grandes clubes deportivos de uso privado (golf, estadios, piscinas, etc.). Un nuevo tipo aparecido en los noventa en nuestro país son los Parques Temáticos de explotación privada, que en la mayor parte de los casos se desarrollan en suelo público y con inversión pública (Warner Bros en Madrid, Terra Mítica en Benidorm e Isla Mágica en Sevilla), salvo Port Aventura en Tarragona, desarrollado por una empresa privada aunque con fuerte apoyo de la Generalitat.

\section{E. Grandes acontecimientos mundiales: deportes, expos, ferias comerciales, etc.}

Los dos grandes ejemplos españoles son la Olimpiada de Barcelona y la Expo de Sevilla, celebradas ambas en 1992. (MOPU, 1985; Urbanismo, 1987; BusQueTs, 1992). En Sevilla se creó una empresa estatal que en colaboración con el Ayuntamiento (con un plan urbanístico en elaboración) realizó todas las obras e infraestructuras de la isla de la Cartuja, así como la coordinación de actuaciones viarias, ferroviarias (Alta Velocidad con Madrid) y aeroportuarias, que supusieron la transformación de importantes elementos estructurales, y de distintos barrios, de la ciudad. En Barcelona se creó un Holding entre el Ayuntamiento y el Gobierno central, con el que colaboró el Gobierno regional mediante convenios, que se ocupó asimismo de la reestructuración del viario urbano (B20), nuevas terminales aeroportuarias, torres de telecomunicaciones y la reurbanización de cuatro áreas para instalaciones olímpicas entre las que destaca la de la Villa Olímpica, que supuso la 
sustitución de instalaciones industriales y ferroviarias obsoletas, por una nueva pieza de ciudad multifuncional que abría la ciudad al mar en la costa nordeste.

\section{F. Operaciones de remodelación interna}

Algunas ciudades emprendieron en los ochenta actuaciones de revitalización en barrios degradados del centro de la ciudad. Los más destacables son Valencia, Barcelona y la actuación de San Francisco el Grande en Madrid. Esta última consiste en un buen ejercicio de diseño y de gestión municipal de una zona inacabada del perímetro del casco antiguo (MOPU, 1985). En los casos de Barcelona y Valencia la actuación abarca el conjunto del casco antiguo, y se ha ido haciendo progresivamente más integral con la incorporación de actuaciones físicas de esponjamiento y reordenación de los espacios públicos y el tráfico, para incorporar después medidas sociales y de empleo. En Valencia se creó una oficina municipal que ha dirigido el proceso realizando convenios con distintos agentes para actuaciones concretas, y en Barcelona se creó una empresa y un Consejo del Área de rehabilitación integral, con participación empresarial y vecinal, para controlar el desarrollo de la operación $(\mathrm{CNH}$, 1996b). El caso más relevante de remodelación de zonas periféricas se dio en Madrid en donde se construyeron en los ochenta 40.000 viviendas para realojo en barrios desfavorecidos, y en especial en Vallecas (sudeste de Madrid), donde además de los barrios de remodelación el Consorcio que creó la Comunidad completó posteriormente la trama urbana con el nuevo barrio de Madrid Sur con 7.000 viviendas, edificado por cooperativas (CNH, 1996b).

\section{G. Nuevos ensanches de la ciudad}

Un antecedente de gran interés es el rediseño que la Comunidad de Madrid tuvo que realizar a mediados de los ochenta de la nueva ciudad de Tres Cantos (norte de Madrid), única nueva ciudad española, que se había iniciado diez años antes por una empresa pública y que se encontraba estancada a medio hacer y sin perspectivas en el mercado inmobiliario. En la reordenación se introdujo una nueva trama para la edificación con parcelas para las nuevas demandas de actividad económica y variedad tipológica residencial, disminuyendo la densidad y promoviendo la calidad urbana y de diseño. La Comunidad de Madrid se caracterizó desde su creación en 1983 por la promoción de suelo para vivienda barata y el apoyo a las cooperativas, destacando entre sus actuaciones la de Valdebernardo, que promovió directamente el Gobierno regional (EzQuiAGA, 1990) y los nuevos barrios promovidos por Consorcios de la Administración regional y los ayuntamientos (CM, 1995).

\subsection{La evaluación de las grandes actuaciones}

Si bien está por hacer una evaluación comparada de las grandes actuaciones, una exposición sobre las mismas no debe dejar de enunciar algunos criterios que deben tenerse en cuenta, tanto para el diseño de las actuaciones como para la valoración de sus impactos una vez realizadas.

Las grandes operaciones tienen un enorme potencial para el gobierno del territorio (ARIAS, 1991), dado que tendrán importantes impactos funcionales, socioeconómicos y ambientales en su entorno, y que las rentas de posición que materializan pueden utilizarse de distinta forma, con distintos efectos redistributivos, según quiénes sean los agentes promotores que se las apropien y cuáles sean sus objetivos.

La definición de las operaciones debe formar parte de una reflexión estructural y estratégica sobre la ciudad, explicitando las razones de su ámbito espacial y su propuesta de desarrollo temporal, en función de los procesos urbanos que se deseen impulsar o fortalecer (provisión de ciertas actividades o servicios, diversificación y complejidad interna, capacitación de los agentes empresariales y el capital humano, mejora de la sustentabilidad urbana, potenciación de la organización de la sociedad civil, etc.). Para ello es necesario definir adecuadamente, en función de los efectos deseados, los contenidos de la actuación: nuevos usos, recualificación de actividades existentes, fortalecimiento de tejidos y redes, etc.

La concertación de la actuación y de la forma de ejecución, con distintos agentes, influirá en la definición de cuáles serán sus contenidos y efectos. Las grandes actuaciones suelen plantearse a través del debate de expertos, y buscando el consenso entre los poderes fácticos y las instituciones, pero suelen evitar a muchos grupos sociales, en particular a los más desfavorecidos, cuyos intereses se consideran suficientemente representados por los gobiernos municipales, y garantizados (seguridad jurídica) por los procedimientos administrativos que se deban cumplimentar a posteriori en el desarrollo de las operaciones, según establezca la legislación aplicable.

Normalmente, los poderosos negocian sus intereses, para mejorar sus retornos en el desarrollo de la operación, desde el origen de las actuaciones, en tanto que los ciudadanos de a pie casi no se enteran de lo que va a ocurrir hasta que no se empiezan a ejecutar.

En las grandes operaciones no se debate a priori suficientemente, y con todos los grupos afectados, sobre los temas de interés general que deberían formar parte, explícitamente, de los objetivos urbanos que han de servir para su desarrollo y evaluación (una cosa son los supuestos previos y otra los efectos a medio y largo plazo). 
Los temas a debatir en cada caso deberían considerar, en relación a la ciudad en su conjunto, el entorno próximo y el propio área de la actuación, al menos los siguientes temas:

- Accesibilidad, conectividad general, telecomunicaciones, red de transporte público, permeabilidad, etc.

- Espacios públicos internos, servicios al entorno, redes de espacios abiertos, etc.

- Actividades incluidas en la operación, adecuación del espacio creado, oportunidad de la escala, densidad, diversidad y complementariedad de las mismas, y con las áreas adyacentes, $\mathrm{u}$ otros polos en competencia, etc.

- Incidencia en la creación de empleo, capacidad multiplicadora, destrucción de empleo en otras áreas y sectores, oferta a grupos desfavorecidos, oportunidades creadas para el desarrollo local en otras zonas conectadas, etc.

- Influencia en la polarización socioeconómica de la ciudad y del entorno, incidencia en las rentas del suelo, procesos de sustitución de residentes (hogares, pequeñas empresas, etc.), oportunidades creadas para el desarrollo comunitario, etc.

- Huella ambiental de la actuación (energía, agua, residuos, etc.) y medidas para la reducción de impactos en la insostenibilidad local y global.

\subsection{La mejora del tejido urbano de la ciudad consolidada como actuación estratégica}

En el contexto actual de transformaciones sociales y económicas y ante objetivos de desarrollo sustentable (calidad de vida, cohesión social, sostenibilidad ambiental, revitalización de una democracia participativa y cooperativa, etc.), también deben considerarse operaciones estratégicas las intervenciones distritales (barrios), que incluyen actuaciones difusas y extensivas para la mejora de la calidad de la ciudad existente, articulando pequeñas actuaciones de relleno, creación de diversificación social, desarrollo local, mejora ambiental, etc., con otros programas sociales y económicos.

Las experiencias piloto de la Unión Europea y las directrices de la Agenda 2000 apoyan esta línea de financiación de actuaciones urbanas (Presidencia de la UE, 1995, 1997, 1998; CCE, 1996, 1998) y también algunas políticas nacionales de Estados miembros (ARIAS, 1996; JACQUIER, 1995), siendo destacables las experiencias francesas (DIV, 1996; MES, 1998), británicas (DETR, 1997, 1998) y holandesas (PeLTENBourg, 1997).

Las actuaciones que se están promoviendo abordan, mediante un único Programa integrado de barrio o distrito, las acciones específicas que requiera cada caso: urbanismo, vivienda, movilidad, revitalización económica, promoción cultural y social, equipamiento y seguridad ciudadana, etc.

Estas operaciones incluyen, según las necesidades de cada área, actuaciones de varios tipos:
- Programas integrados de regeneración urbana, que apoyan la integración social, acogida de inmigrantes, adaptación de los servicios públicos a las nuevas necesidades (hogares unipersonales, ancianos, jóvenes con precariedad, inmigrantes de otras etnias, lengua, etc.), realojamiento y rehabilitación pública de viviendas y edificios en peores condiciones, ayuda a la rehabilitación privada de viviendas (incluyendo la adaptación a nuevas necesidades y las mejoras de sostenibilidad), mantenimiento de actividades económicas tradicionales, y apoyo al desarrollo de pequeñas empresas que faciliten empleos locales (no sólo servicios personales, también otras con alto valor añadido), incrementando la diversidad de usos y actividades, etc. De aplicación, fundamentalmente, en las áreas de edificación antigua.

- Programas de desarrollo local, que aplican las políticas de empleo y fomento de la actividad económica en los barrios, teniendo en cuenta sus oportunidades específicas (PYMES, comercio, áreas industriales, construcción, rehabilitación de edificios, servicios de proximidad, etc.) y las características de su población (cualificación, demanda de servicios, horarios, etc.); facilitando formación, apoyo a los emprendedores, acceso al mercado de trabajo, etc., a través de agencias de desarrollo vinculadas a las PYME y los proyectos de economía social de la zona. De aplicación, fundamentalmente, en los barrios desfavorecidos periféricos de las ciudades con alto nivel de paro.

- Planes de actividad económica, que impulsan centralidades locales, ejes comerciales y áreas de actividad económica (modernización de polígonos, creación de locales para PYMES, etc.), incluyendo la reutilización de instalaciones de servicios y actividades obsoletas o innecesarias (Ferrocarriles, militares, industrias abandonadas, etc.) para reequipar los barrios y generar empleo. De aplicación, fundamentalmente, en barrios y distritos periféricos.

- Planes de movilidad por barrios, que abordan actuaciones de apoyo a la fluidez del transporte en superficie, mejora de la accesibilidad peatonal (aceras, eliminación de obstáculos, etc.), eficiencia del sistema de transporte público (mejora del intercambio en las paradas de transporte, carriles exclusivos en tramos congestionados, etc.), control del tráfico en determinadas calles y zonas, regulación de la distribución de mercancías para que sea eficaz, racionalización del aparcamiento (gestión común de los aparcamientos subterráneos y en superficie) para facilitar el aparcamiento de residentes, etc.

- Programas de reequipamiento que reconsideran las necesidades de equipamientos de los barrios (escuelas infantiles, educación permanente, formación ocupacional, salud, tercera edad, inmigrantes, cultura, etc.), incluyendo aspectos sobre las formas de gestión y horarios, para lograr un mejor servicio y una mayor eficacia en las prestaciones.

- Planes de mejora del medio ambiente urbano que desarrollan medidas de paisajismo, ampliación y sostenibilidad de los 
espacios públicos, para aprovechar las condiciones climáticas, ampliar la extensión de la superficie vegetal y promover la utilización de especies autóctonas, así como la mejora de la eficiencia ambiental de los edificios y la gestión de la demanda de recursos (energía, agua, residuos, etc.).

- Actuaciones de integración social de colectivos específicos. Las políticas sectoriales de servicios sociales para los grupos especialmente vulnerables pueden territorializarse más fácilmente en los barrios desfavorecidos en que se concentran las necesidades, integrando las políticas sociales con otras actuaciones en el marco de Programas de Regeneración en los barrios desfavorecidos, ya que en ellos hay mayor densidad y superposición de actuaciones (salarios de integración, pensiones, desempleo, salud, formación, asistencia, etc.).

\section{La concertación de actuaciones urbanas}

Se ha expuesto que las actuaciones urbanas tienden a ser complejas en sus contenidos y estratégicas en su incidencia en la ciudad. Se trata de actuaciones que plantean un paquete de políticas complementarias, incluyendo medidas infraestructurales, urbanísticas, ambientales, sociales y de actividad económica, aunque no suelen utilizar adecuadamente un enfoque integrado que parta del análisis de los problemas desde el territorio y con los afectados, quedando sesgadas hacia la infraestructura y lo inmobiliario.

Este tipo de políticas urbanas multisectoriales que implican a varios Organismos requiere la coordinación de los distintos agentes sectoriales que han de participar en las operaciones, y el desarrollo de procedimientos y mecanismos de concertación, tanto en la concepción como en la ejecución de las actuaciones, mediante la cooperación de todos los agentes implicados, sean ejecutores o afectados.

La primera concertación necesaria es la de las Administraciones públicas que tienen competencias concurrentes en las actuaciones. Sólo a partir de esta base, de «certidumbre» en cuanto a la existencia de una iniciativa pública coordinada, se podrá incorporar a las actuaciones a otros agentes como los organismos internacionales con sus programas de financiación, la iniciativa privada, u otros sectores de la sociedad civil que puedan resultar necesarios para su mejor desarrollo (Organizaciones sin ánimo de lucro, Organizaciones vecinales o locales, etc.).

La concertación facilita la cofinanciación desde las distintas Administraciones y la financiación vía sector privado, mediante activos inmobiliarios existentes o aprovechamientos urbanísticos que se generen en las actuaciones, y también por cánones en concesiones de servicios, que son los mecanismos habitualmente utilizados en nuestro país.

Para desarrollar una política de actuaciones concertadas, es necesario que las Administraciones dispongan de un procedimiento para la identificación, definición e instrumentación de este tipo de actuaciones urbanas, evitando mecanismos discrecionales y aleatorios que dejen las operaciones en manos de intereses conflictivos con las necesidades del conjunto de la ciudad. Un procedimiento que permita la coordinación de los agentes desde el origen de las actuaciones, contemplando la ciudad, y el barrio, como sujetos activos, y no como objetos indirectos de las consecuencias de las políticas sectoriales.

\subsection{Procedimiento de concertación: fases y funciones a desarrollar}

La concertación de actuaciones urbanas en nuestro país se ha producido fundamentalmente en operaciones vinculadas al desarrollo o integración de infraestructuras en las ciudades y a la urbanización de suelo residencial o empresarial y áreas de rehabilitación de vivienda, según se ha expuesto en los puntos anteriores.

A mediados de los ochenta se inició un nuevo período en la concepción de la concertación urbanística en España, destacando la nueva Política territorial de la Comunidad de Madrid iniciada en 1983, las acciones emprendidas para la Olimpiada de 1992 en Barcelona (aprobada por el COI en 1986) y la puesta en marcha de la EXPO 92 en Sevilla. Los procedimien. tos utilizados fueron sustancialmente distintos en las tres ciudades:

- En Barcelona se crea en 1986 el Holding Olímpico (HOLSA) público, que comienza a desarrollar y ejecutar los proyectos de ciudad que se abordan con motivo de este evento, realizándose en el período 1988-1991 el primer Plan estratégico de la ciudad, que complementa las actuaciones en realización con otras medidas y mejora el apoyo social a los procesos en marcha.

- En Sevilla se crea la Sociedad Estatal Expo 92, que se apoya en el Plan Urbanístico que se terminó en esas fechas, y que desarrolló y ejecutó los proyectos vinculados a la Exposición universal.

- En Madrid se crearon desde 1983 diversos instrumentos de actuación (ARIAS, 1989) con una activa creación de Consorcios (Transporte regional, Pasillo Verde Ferroviario, Parques empresariales, Nuevos barrios, etc.), elaborándose en 1987 la primera estrategia territorial subregional (ARIAS, 1991).

El Plan Director de Infraestructuras de 1993 por el MOPTMA puso en marcha un procedimiento de concertación en las ciu- 
dades para Actuaciones estratégicas de Transporte y Urbanismo, actuaciones en Infraestructuras (Planes Intermodales de Transporte, Red Viaria y Ferrocarril) y mejora del Medio Ambiente Urbano (MOPT, 1993; Ciudad y Territorio, 1993b).

Las Actuaciones estratégicas se propusieron a lo largo de los siguientes cuatro años en 20 ciudades (Estudios Territoriales, 1992), poniéndose en marcha, además del Consorcio del Pasillo Verde Ferroviario de Madrid, el de Jerez, las Sociedades de Bilbao Ría 2000 y de Cinturón Verde de Oviedo y las actuaciones de Cádiz, Plataforma Logística del Llobregat y Puerto Seco de Madrid. En 1997 el nuevo Gobierno del Partido Popular no continuó este programa, aunque siguen ejecutándose las actuaciones que estaban en marcha y se firmó el convenio de actuación en el municipio de Cádiz.

Desde los ochenta se empezaron a aplicar los programas sociales de la Unión Europea, extendiéndose en 1995 una nueva fase de la concertación de actuaciones integrales para la regeneración de áreas urbanas (ARIAS, 1998b) al aplicarse la iniciativa URBAN de los Fondos Estructurales Europeos.

A partir de todas estas experiencias se pueden resaltar algunas cuestiones importantes para la concertación de actuaciones urbanas:

\section{A. Definición de programas-marco}

Las actuaciones de mediados de los ochenta de Madrid, Barcelona y Sevilla responden a programas urbanísticos o territoriales, específicos.

Ya en los noventa, los programas de actuación que se han venido aplicando se definieron inicialmente en el Plan Director de Infraestructuras (MOPT, 1993; Ciudad y Territorio, 1993b), así como en otros planes nacionales y regionales (ARIAS, 1998b), como los Planes de Vivienda (Áreas de rehabilitación integral ARI), Planes de Acción Social, y programas europeos (Iniciativa URBAN, Proyectos Piloto, Horizon, WHO, LIFE, etc.).

La definición de estos programas parte de la detección de problemas tipo, a partir de estudios comunitarios, nacionales, regionales y locales que valoran la magnitud de los problemas, las posibles formas de actuación, la experiencia de actuaciones piloto, etc. (ARIAS, 1998b), aunque no se suele explicitar la experiencia y estudios en que se fundamentan los programas. Cada programa establece unos criterios y procedimientos de elegibilidad para identificar y programar actuaciones.

En España existe una gran carencia en materia de Observatorios de información y diagnóstico, necesarios para facilitar estas tareas, en los que deberían colaborar las Administraciones con otros agentes implicados.

\section{B. Identificación de actuaciones}

Dada la complejidad de los problemas y de las intervenciones necesarias, la aplicación de los criterios de elegibilidad para programar actuaciones no puede ser exclusiva de una Administración, ni de un Departamento sectorial, y suele requerir la concertación entre Administraciones. En general, este tipo de programas genera órganos especializados de concertación en las Administraciones responsables, es decir, un interlocutor que se ocupa de la coordinación interna de todas las competencias de esa Administración, y cuya mera existencia expresa: la voluntad política de concertar competencias (sectoriales, territoriales y urbanísticas) y la necesidad de cofinanciar las actuaciones, así como la disponibilidad a facilitar la realización de un esfuerzo conjunto en la obtención de recursos extrapresupuestarios y de financiación europea.

El principal criterio de selección en los programas derivados del PDI fue la viabilidad de los proyectos que se prepararon para las líneas elegibles de actuación, viabilidad que se basó en la voluntad de cooperación de las Administraciones para facilitar: la contención del gasto en las propuestas de intervención, y la aportación de fondos presupuestarios y de las disponibilidades extrapresupuestarias (edificabilidad, concesiones de explotación, etc.) propias de cada operación. En las ARI la identificación de actuaciones ha dependido de la concertación de las tres Administraciones para la asignación de los recursos regionalizados del Plan de Vivienda a áreas de necesidad, creándose instrumentos ad boc de actuación, mientras que en los URBAN se han seleccionado las actuaciones, a través de un concurso, sobre la base de los objetivos de regeneración urbana propuestos por la UE, y las garantías de cooperación y financiación de todos los agentes.

\section{Formulación de los proyectos técnicos y de la instrumentación de la ejecución}

Esta fase de la concertación tiene que hacerse de forma conjunta entre los futuros cooperantes en la ejecución (Estudios Territoriales, 1992; ARIAS, 1999b). Las fórmulas habituales aplicadas son los Convenios para la definición de los proyectos, para lo que se establecen las oportunas Comisiones de Seguimiento (Pasillo Verde Ferroviario de Madrid, Delta del Llobregat y Bahía de Cádiz), o la creación de una sociedad instrumental que en su primera fase de trabajo realiza los proyectos y los estudios de viabilidad (Bilbao Ría 200, S. A.; C. V. Oviedo, S. A.). En áreas de rehabilitación, URBAN, etc., la iniciativa de la formulación de los proyectos suele centrarse en las Administraciones Territoriales, especialmente la local, si bien realizando convenios con otros agentes para su planteamiento y desarrollo, que pueden llegar a la utilización de sociedades mixtas con representación de los distintos agentes (Ciutat Vella 
en Barcelona, o la participación de Bilbao Ría 2000, S. A., en el URBAN de Baracaldo).

En diversos países europeos es habitual la participación de la Administración Central en la concertación de actuaciones urbanas (ARIAS, 1996), coordinando sus inversiones en infraestructuras así como fondos para vivienda, medio ambiente urbano o políticas sociales y de empleo, como ocurre en Francia (Contratos de Ciudad), Holanda, Alemania, Reino Unido (Presupuesto único de regeneración), etc.

Sin embargo, suele echarse en falta, especialmente en España y sobre todo en los grandes proyectos urbanos, la participación del sector local, limitándose la cooperación a los agentes con competencias administrativas o capacidad inversora. En otros casos alguna Administración no se asocia plenamente por recelos políticos. Ambas situaciones pueden ser motivo para que una operación tarde en arrancar más de lo debido o incluso para que no llegue a ponerse en marcha.

\section{Gestión y ejecución}

La compleja imbricación de competencias en la ejecución de las actuaciones y las difíciles fórmulas de cofinanciación y de control de la utilización de los recursos extrapresupuestarios, han dado lugar a la búsqueda de mecanismos de gestión ad boc, como pueden ser los Consorcios o las sociedades instrumentales (sociedades anónimas participadas por los agentes concertados). Si las sociedades se han creado con anterioridad para realizar los estudios de viabilidad (punto c), en su segunda fase de trabajo pueden pasar a desarrollar las actuaciones (Bilbao y Oviedo). En otros casos, una vez concluidos los estudios de viabilidad por la Comisión de seguimiento del Convenio, las Administraciones acuerdan la creación de Consorcios ( $\mathrm{Pa}$ sillo Verde Ferroviario de Madrid o Jerez). La Comunidad de Madrid ha desarrollado desde mediados de los ochenta una gran experiencia de fomento de Consorcios interadministrativos en la urbanización de suelo (CM, 1995).

El control de la actividad de los Consorcios y Sociedades en que participa el Ministerio de Fomento se realiza a través de los Consejos de Administración de las mismas. En las ya creadas participa el Ministerio de Fomento (1 o 2 Consejeros por Sociedad) a través de sus empresas públicas (SEPES, REN. FE, FEVE, AENA y Autoridades Portuarias) que disponen al menos de un $50 \%$ de los consejeros, y desembolsan en conjunto una proporción equivalente del capital social inicial de lanzamiento de las empresas.

Las ARI disponen de una Comisión Gestora específica para el control de la actuación en la que participan todas las Administraciones y otras organizaciones, y los proyectos URBAN constituyen normalmente un Órgano gestor ad hoc para la dirección y coordinación de todos los proyectos.

\section{E. Financiación}

En las actuaciones vinculadas a obras de infraestructuras la concertación financiera se establece a través de los Convenios marco de ejecución de las actuaciones una vez desarrollados y valorados los proyectos y programas a acometer (los Convenios de financiación se producen dentro de las sociedades instrumentales si éstas se hubiesen constituido previamente para la realización de los estudios técnicos).

Los Convenios establecen un marco de financiación global entre los socios que contempla las aportaciones a la sociedad del aprovechamiento urbanístico posible en los terrenos de la actuación, así como la aportación presupuestaria complementaria para completar la actuación, que dependerá asimismo de la posibilidad de obtener Fondos Europeos para el Consorcio o Sociedad actuante (ARIAS, 1999b).

La financiación europea se ha producido en las grandes operaciones a través de FEDER (Fondos estructurales) y Mecanismo financiero (EFTA), realizándose las aportaciones directamente a las sociedades instrumentales (Bilbao, Oviedo y Jerez).

La financiación a través de recursos extrapresupuestarios derivados de aprovechamientos urbanísticos se aplica en todas las actuaciones, pero de momento sólo se ha aproximado a la autofinanciación (coste total de la operación) en el Pasillo Verde Ferroviario de Madrid. Normalmente las distintas Administraciones se hacen cargo de algunas obras complementarias a través de sus presupuestos ordinarios. La incorporación de financiación privada se produce a través de la adquisición del suelo edificable y la consiguiente construcción de edificios de uso privado, o de la aportación de parte de los beneficios de explotación de la concesión de bienes inmuebles (Operación Chamartín en Madrid).

Los programas ARI o URBAN disponen de fondos propios. En los URBAN los fondos europeos suponen tan sólo el 50\% del conjunto, pudiendo incorporarse financiación extrapresupuestaria, junto a las aportaciones de distintas organizaciones y a las presupuestarias de las Administraciones que participan en la actuación. Las ARI suelen complementar la inversión en rehabilitación de vivienda con otras inversiones en desarrollo de planes especiales, incluyendo vivienda nueva, urbanización, medio ambiente urbano, transporte o actuaciones sociales y de empleo.

\section{F. Seguimiento y evaluación}

Además del seguimiento del desarrollo material de las actuaciones, se debe mantener un seguimiento y evaluación de sus impactos urbanos, si bien las experiencias españolas se 
muestran muy deficitarias en esta tarea que, sin embargo, es fundamental para el futuro. Ambas formas de seguimiento deben realizarse por las propias sociedades o consorcios, con el consiguiente control por cada Administración en sus niveles de competencia.

Por parte del M. de Fomento el seguimiento del desarrollo de la actividad de las Sociedades y Consorcios, venía realizándose a través de la antigua D. G. de Actuaciones Concertadas en las Ciudades y de SEPES (presente en todas las sociedades instrumentales), con independencia de los controles de la Intervención General de la Administración del Estado.

Sin embargo, es necesario, además, realizar un seguimiento global de los programas de actuaciones concertadas y de los resultados comparados de las actuaciones en ejecución a través de los Observatorios de información y diagnóstico, como los descritos en el punto A (ArIAS, 1997).

* Director Técnico. Dirección General de Programación Económica. Ministerio de Fomento.

\section{Bibliografía}

AceBillo, J. A. (1992), «El progresivo cambio de escala en las intervenciones urbanas de Barcelona», revista Urbanismo, núm. 17: 10-25, Madrid: C. O. Arquitectos y Segundo Congreso Internacional de Ordenación del Territorio, Universidad Politécnica de Valencia.

ARIAS GoYTRE, F. (1989), «Las estrategias territoriales de ámbito subregional», revista Urbanismo, núm. 8: 45-62, Madrid: C. O. Arquitectos.

- (1991), «¿Ordenación o política territorial?», Segundo Congreso Internacional de Ordenación del Territorio, Universidad Politécnica de Valencia.

- (1996), «La política de ciudades de los gobiernos nacionales», Cuadernos económicos de Granada.

- (1997), «La estructura urbana y el régimen de suelo como bases de la sostenibilidad y la cohesión social», Jornadas sobre el nuevo marco de la regulación urbana, revista Urban, ETSAM, 1998.

- (1998a), «Politicas de sostenibilidad en España, las ciudades medias», VII Semuna de estudios urbanos, Universidad de Lleida.

- (1998b), «La desigualdad urbana en España», Documento de trabajo, M. de Fomento.

- (1998c), «Política de ciudades: oportunidades de la actuación estatal», Seminario «La ciudad futura: transformaciones territoriales y políticas urbanas en Europa», Sevilla: Universidad Internacional Menendez y Pelayo.

- (1999a), «La regeneración de la ría de Bilbao: más allá del Guggenheim», Conferencia en el Seminario sobre experiencias en ambientes urbanos bistóricos, Ay. Málaga.

- (1999b), «Ciudades y Globalización: transformaciones sociales y oportunidades urbanas», Cuadernos económicos de Granada.

Ayuntamiento de Barcelona (1999), Urbanismo en Barcelona, Ayuntamiento de Barcelona.

Borja, J., y Castells, M. (1996), Local y Global: la gestión de las ciudades en lo era de la información, UNCHS, Taurus, 1997.

BusqueTs, J. (1992), Barcelona, evolución urbanística de una capital compacta, Madrid: MAPFRE ed.

CASTELLS, M. (1997), La era de la información: economia, sociedad y cultura, 3 vol., Madrid: Alianza.

CiUdad y Territorio (1993b), Perspectivas en torno al Plan Director de Infraestructuras, numero monográfico, revista Ciudad y Territorio-Estudios Territoriales, núm. 97, Madrid: Ministerio de Obras Públicas y Transportes.
Comisión de las Comunidades Europeas (CCE) (1990), Libro verde del medio ambiente urbano, COM (90) 218, Publicaciones de la Comunidad Europea.

- (1996), Ciudades europeas sostenibles, Grupo de expertos sobre medio ambiente urbano. Disponible en http://europa.eu.int/comm/environment/urban/home _en.htm.

- (1998), Marco de actuación para una politica urbana sostenible en la Unión Europea, COM (98) 605 Final. Disponible en http://www.inforegio.cec.eu.int/urban.

Comité Nacional de Hábitat II (CNH) (1996a), Agenda Hábitat España: documento de trabajo, Centro de Publicaciones, Ministerio de Fomento. Disponible en http://habitat.aq.upm.es.

- (1996b), Primer Catálogo de Buenas Prácticas, Centro de Publicaciones, MOPT. MA. Disponible en http://habitat.aq.upm.es.

- (1999), Segundo Catálogo de Buenas Prácticas, Centro de Publicaciones, MOPT. MA. Disponible en http://habitat.aq.upm.es.

Comunidad de MadRId (CM) (1995), Los Consorcios urbanisticos en la Comunidad de Madrid: una fórmula de gestión de suelo para los años noventa, Madrid: Consejería de Política Territorial.

Chanan, G. (1997), Active Citizenship: Getting to the Roots, European Foundation for the Improvement of Living and Working Conditions, Dublin: Publicaciones de la Comisión Europea.

Delegation Interministerielie a la Ville (DIV) (1996), Les contrats de Ville, Paris: DIV.

Department of the Environment, Transport and Regions (DETR) (1997), Involving Communities in Urban and Rural Regeneration: a guide for practitioners, London: DETR.

- (1998), «Community - Based Regeneration Initiatives», Working paper, Lon. don.

Estudios Territoriales (1992), «Una política para las ciudades», número monográfico, revista Estudios Territoriales, núm. 39, Madrid: Ministerio de Obras Públicas y Transportes.

EzQUiAGA, J. M. (1990), «PAU de Valdebernardo: inventar el espacio periférico», revista Geometría, núm. 9: 34-41, Málaga. 
- (1993), «Madrid: nuevos instrumentos de política territorial», revista Ciudad y Territorio-Estudios Territoriales, núm. 95-96: 107-122, Madrid.

FerRán AlFaro, C. (1991), «El efecto territorial de los grandes proyectos urbanos», Segundo Congreso Internacional de Ordenación del Territorio, Universidad Politécnica de Valencia.

Geddes, M. (1998), Local Partnership: A Successful Strategy for Social Cobesion?, European Foundation for the Improvement of Living and Working Conditions, Dublin: Publicaciones de la Comisión Europea.

Hernández Aja, A.; Alguacil, J.; Medina, M. J., y Moreno, C. (1997), La ciudad de los ciudadanos, Madrid: Ministerio de Fomento.

JACQUiER, C. (1995), Les approches intégrées de développement urbain: conclusions, propositions et recommendations, Quartiers en Crise programme, Brussels: Association Internationale pour la Revitalisation des Quartiers en Crise.

Jewson, N., y MacGregor, S. (1997), Transforming Cities: contested government and new spatial divisions, London: Routledge.

LeIRA, E. (1984), «Del Plan de Madrid: elementos para un debate», revista Ciudad y Territorio, núm. 59-60: 81-107, Madrid: Instituto de Estudios de Administración Local.

Ministère de L'Emploi et DE la Solidartté (MES) (1998), Demain, La Ville: 50 propositions pour l'avenir des villes, Informe de JP Sueur, Alcalde de Orléans, Paris: Ministère de L'Emploi et de la Solidarité

MOPT (1993), Plan Director de Infraestructuras 1993-2007, Secretaria General de Planificación y Concertación Territorial.

MOPU (1985), Grandes proyectos urbanísticos, Madrid: Instituto del Territorio y el Urbanismo, Ministerio de Obras Públicas y Urbanismo.

- (1990), 10 años de planeamiento urbanistico en España, Madrid: Instituto del Territorio y el Urbanismo, Ministerio de Obras Públicas y Urbanismo, Madrid.

NAREDo, J. M. (1996), «Sobre la insostenibilidad global de las actuales conurbaciones y el modo de paliarla», en Primer Catálogo español de buenas prácticas Hábitat II, M. de Fomento. Disponible en http://habitat.aq.upm.es.

OCDE (1996), Innovative policies for sustainable urban management: The Ecological City Project, Final report, Urban Affairs Division. OCDE Paris.

- (1998), Integrating Distressed Urban Areas, Final report, Urban Affairs Division, OCDE Paris.
Organización de Naciones Unidas (ONU) (1992), Programa 21: Declaración de Rio sobre el Medio Ambiente y el Desarrollo, Cumbre mundial sobre el Medio Ambiente y el Desarrollo, PNUMA, Oficina de Publicaciones de Naciones Unidas.

- (1995), Declaración de Copenhague sobre Desarrollo Social y Programa de Acción, Cumbre mundial sobre Desarrollo Social, PNUD, Oficina de Publicaciones de Naciones Unidas.

- (1996), Programa de Hábitat II: Declaración de Estambul sobre los Asentamientos Humanos, Cumbre mundial sobre los Asentamientos Humanos, CNUAH, Oficina de Publicaciones de Naciones Unidas.

Peltenbourg, M. (1997), The Deventer Neighbourbood Approach, Raster Foun dation. Disponible en http://www.raster-groep.nl.

PraTs, F. (1996), «Sostenibilidad y políticas urbanas y locales en las ciudades españolas», en Primer Catálogo español de buenas prácticas Hábitat II, M. de Fomen to. Disponible en http://habitat.aq.upm.es.

PresidenCia de la Unión EuRopea-Francia ( 1995a), Zonas urbanas y cobesión social en Europa: ¿qué acción para las ciudades?, Paris: Délégation Interministerielle à la Ville.

Presidencia de la Unión EuRopea-España (1995b), Ciudades: espacios de problemas y oportunidades, Madrid: Ministerio de Obras Públicas, Transportes y Medio Ambiente.

Presidencia de la Unión Europea-Holanda (1997), National Urban Policy in the European Union, Rotterdam: European Institute for Comparative Urban Research, Erasmus Universiteit

PresidenCia de la Unión EuRopea-Reino Unido (1998), Urban Exchange Initiative: Improving Good Practice in Urban Development in the European Union, Lon don: Department of the Environment, Transport and Regions.

RodRiguez Villasante, T. (1996), «Participación e integración social», en Primer Catálogo español de buenas prácticas Hábitat II, M. de Fomento. Disponible en http://habitat.aq.upm.es.

RUEDA, S. (1996), «La ciudad compacta y diversa, frente a la conurbación difusa», en Primer Catálogo español de buenas prácticas Hábitat II, M. de Fomento. Disponible en http://habitat.aq.upm.es

URBANISMo (1987), «Madrid, Barcelona, Sevilla: las realizaciones», revista Urbanismo, núm. 17, Madrid: C. O. Arquitectos.

-- (1989), «El Plan General de Madrid cuatro años después», número monográfico, revista Urbanismo, núm. 7, Madrid: C. O. Arquitectos. 
